\title{
Skill Variety, Family-friendly and Task Performance: The Moderation of Market Orientation
}

\author{
Lu Gao ${ }^{\text {a }}$, Zhihua Lian ${ }^{\mathrm{b}, *}$ \\ Xiamen University Tan Kah Kee College, Zhangzhou 363105, China \\ agaolu@xujc.com, ${ }^{\mathrm{b}}$ Corresponding Author:lawrancelian@xujc.com
}

Keywords: Family-friendly, Skill variety, Market orientation, Task performance.

\begin{abstract}
This study explored relations of skill variety, family-friendly, market orientation and task performance among 425 Chinese new generation employees. Results indicated has a significant positive impact on skill variety and task performance, family friendly plays a mediating role between skill variety and task performance. In addition, market orientation makes a moderated effect between skill variety and task performance. The research verified the data does not exist problems of common methods biases by the proposed SEM.
\end{abstract}

\section{Introduction}

"The world of work is now different than it was then, perhaps fundamentally so." Said Oldham and Hackman (2010). Indeed, no matter the business culture of company, the management policy or the employees' job characteristics are changing under the current uncertain and competitive business environment. In a competitive market, more and more companies find that the degree of an organization orientation, such as market orientation, become crucial for them to stay competitive (Chin, Lo, \& Ramayah, 2013). Many current job require employees to master more skills continuously (e.g., new knowledge, interpersonal skills, cognitive skills) to keep pace with the increasing competitive demands (Wegman et al., 2016). The increasing demographic and social changes result in increasing work and family conflict, which in urgent need of some management policy, such as family friendly, to deal with it. Accordingly, skill variety, family friendly and market orientation as the popular words in modern management field, are received extensive attention of society and mentioned by a number of literatures (e.g., Hackman, \& Oldham, 1975; Demerouti, 2006; Chen, Shin, \& Yeh, 2011; Mauno, \& Ruokolainen, 2015; Caillier, 2016). According to Hackman and Oldham (1975), "Skill variety" is the first dimension of the job characteristic model, which is defined as "the degree to which a job requires a variety of different activities to carry out the work, which involve the use of a number of different skills and talents of the employee". Majority of past studies only discussed how the whole job characteristic impact on employees' behaviors (e.g., Daniels, 2006; Chu, \& Lai, 2011), while there is almost no literature focused on the individual influence of skill variety on employees' behaviors. On the other hand, family friendly refers to "any benefit, working condition, or personnel policy to assist family members in balancing the conflicting demands of work and home life" (Glass, \& Fujimoto, 1995), which is generally used to support employees to balance the conflicting demands of work and family life (Pradhan, Jena, \& Kumari, 2016). In early studies, family friendly is usually used as an antecedent to describe the influence of work family fit on employees' favorable behaviors and we regret to find that it seldom appears as mediating variable. Thus, compared with previous research, we choose different point of view to investigate how the skill variety individually lead to favorable employees' behavior like better task performance and how the family friendly mediates their relationship. Besides these, we simultaneously examine the moderating role of market orientation to find whether the magnitude of relationship between skill variety and task performance is different under this environment. 


\section{Literature Review and Hypotheses}

\subsection{Skill Variety}

Hackman and Oldham (1975) introduced the first version of Job Characteristics Model and provided measures of the five core dimensions, which are named as skill variety, task identity, task significance, autonomy and feedback separately. Among them, "task identity" refers to "the degree to which the job requires completion of a 'whole' and identifiable piece of work-that is, doing a job from beginning to end with a visible outcome". "Task significance" refers to "the degree to which the job has substantial impact on the lives or work of other people-whether in the immediate organization or in the external environment." "Autonomy" refers to "the degree to which the job provides substantial freedom, independence, and discretion to the employee in scheduling the work and determining the procedures to be used in carrying it out." while "Feedback" refers to "the degree to which carrying out the work activities required by the job results in the employee obtaining direct and clear information about the effectiveness of his or her performance. Besides these, "Skill variety", as the first dimension of the model, is defined as "the degree to which a job requires a variety of different activities to carry out the work, which involve the use of a number of different skills and talents of the employee". Each of these dimensions leads to positive psychological states such as feelings of responsibility and meaningfulness, which in turn incentive employees to perform well in the future (Hackman, \& Oldham, 1975). In our study, we choose "skill variety" as the antecedent to describe the impact of job characteristics on employees' performance. This definition of "skill variety" focuses on the degree to which a job has impact on the work of people, emphasizing the challenge of job rather than individual characteristics (Demerouti, 2006). Chen, Shin and Yeh (2011) argued that skill variety is one critical element of individual creativity, which includes technical skills, expertise and factual knowledge in a given domain, as well as the tacit knowledge concerning the appropriate alternatives, problem-solving approach and the feasible solutions. Moreover, Wegman et al. (2016). et al. (2016) pointed out that mean levels of skill variety perceptions have increased along with the emergence of the knowledge economy and the expansion of employees' roles to keep pace with the increasing competitive demands. There is a greater demand for "soft skills" such as interpersonal skills or cognitive skills. Together, skill variety consists of a number of different activities, work procedures and processes necessary to accomplish a task, which requires employees to seek and use more talents and skills (Noefer, et al., 2009). Also, Skill variety requires that employees do a number of different tasks, keep learning new things and to be very creative, which sometimes may become a stressor for employees. Similarly, the stress may also stem from the role ambiguity and role conflict caused by skill variety when the jobs have considerable variation (Lambert, \& Paoline, 2008).

\subsection{Task Performance}

The definition of job performance means the presentation when employees want to fulfill the regulated or expected role for organizational goal, which also means how much the staffs have done within the organization (Campbell, 1990). In the early studies, scholars explored the job performance construct focused on task requirement. For instance, Fleishman (1975) used four approaches to identify dimensions of job performance, which were behavior description approach, behavior requirements approach, task characteristics approach and abilities approach. And the four approaches were limited to what we now refer to as task performance. After this, Borman and Motowidlo (1993) divided job performance into two categories, named task performance and contextual performance separately. He defined task performance as " the proficiency with which incumbents perform activities that are formally recognized as part of their jobs; activities that contribute to the organization's technical core either directly by implementing a part of its technological process, or indirectly by providing it with needed materials or services". While Contextual performance means the extra-role behaviors engaged in by employees over and above their task requirements, which emphasizes selflessness and mutual cooperation (Chu, \& Lai, 2011). In recent years, the definition of job performance has been expanded to three broad dimensions: task performance, organizational citizenship behavior and counterproductive behaviors. According to Rotundo and Sackett (2002), each of these three dimensions contributed to overall performance ratings and task performance 
occupies the greatest weight. That's why we can see that "task performance" is usually called "core task performance” in a substantial number of literatures (e.g. Rotundo, \& Sackett, 2002; Viswesvaran, \& Ones, 2000). In conclusion, task performance refers to basic required duties and the outcome of job performance related to the mission accomplishment within a particular organization, which is the core dimension of job performance.

\subsection{Skill variety and task performance}

According to Hackman and Oldham (1975), job characteristics could stimulate positive psychological states, which include meaningfulness of work and feelings of responsibility, and in turn incentive employees to perform well in the future. Moreover, skill variety is considered as the main source of meaningfulness of work when employees enjoy a variety of skills in performing the job (Johari, \& Yahya, 2016). Piccolo and Colquitt (2006) proposed that jobs regarded as important, challenging and allows employees to use various skills and talents are more likely to create perceived meaningfulness in employees' job, while the meaningfulness of work could improve employees' job performance because they believe that their job can make significant contributions to the society (Ghosh et al., 2015). Similarly, Chu and Lai (2011) founded that skill variety is significantly positively influence the task performance, because task performance is the core dimension of job performance and occupies the greatest weight of it ( Rotundo, \& Sackett, 2002). Accordingly, we can image that skill variety facilitate employee's task performance positively. Therefore, we hypothesis as follows:

Hypothesis 1: Skill variety is positively related to task performance.

\subsection{Family friendly as a mediator}

The term family friendly generally regarded as "any benefit, working condition, or personnel policy to assist family members in balancing the conflicting demands of work and home life" (Glass, \& Fujimoto, 1995), which may be formal policies or informal emotional or psychosocial support (Mauno, \& Ruokolainen, 2015). The formal policies cover a substantial number of family friendly practical policies. Nowadays, there is no generally accepted list of them, thus the perspective of family friendly are often defined variously by different researchers. For instance, Allen (2001) pointed out that family friendly included flexible work arrangement (e.g., flexible work hours, compressed work weeks) and family care assistance (e.g., child-related benefits). Glass and Fujimoto (1995) divided family friendly into three categories: they were separately leave benefits (e.g., sick leave, paid vacation time, parental leave with job security), dependent care benefits (e.g., child care referral services, subsidies) and work schedule policies (e.g., flexible hours, working part-time, working some hours at home ). Schmidt and Duenas (2002) indicated that"employee assistant programs"were important part of family friendly, which provided intervention for work-related problems or personal problems to assist employees to overcome the obstacles in their work and family lives. These programs consist of parenting, marital, elder care, occupational health audits, tuition assistance, training even health insurance etc. On the other hand, the informal emotional or psychosocial support of family friendly have sometimes been defined as "family-supportive organizational perceptions" or "family friendly supervision”, which pay more attention to support from supervisors and coworkers (Mauno, \& Ruokolainen, 2015). Allen (2001) defined "family supportive organizational perceptions" as "the global perceptions that employees form regarding the extent to which the organization is family-supportive". Wang, et al. (2013) indicated that "family friendly supervision" is the supervisory behaviors such as empathizing, recognizing and identifying with employees' demands to balance work and family life. To sum up,

The combination of formal family friendly policies and informal family friendly supportive supervision lead to a family friendly workplace and employees who work in a family friendly workplace have been found to exhibit a lot of positive behaviors (Foley, Linnehan, Greenhaus, \& Weer, 2006), including greater work commitment, more organizational citizenship behavior and better job performance (Seong, 2016)

Family friendly is usually used as an antecedent to describe the influence of work family fit on employees' favorable behaviors and we regret to find that it seldom appears as mediating variable. However, we found that family friendly could be a mediating variable between skill variety and task 
performance. For instance, skill variety requires employees to learn new things continuously and master a number of different tasks (Lambert, \& Paoline, 2008), while organization can offer more family friendly programs like overseas or domestic on-the-job training to ensure employees' skills variety (Steyn, \& Vawda, 2014). At the same time, family friendly programs like child care and flexible hours enable employees to have much more time to master various skills and the perception of organizational family friendly support can decrease employees' feeling of stress which caused by complicated skilled required (Caillier, 2016). On the other hand, Ko, Hur, \& Smith-Walter (2013) indicated that family friendly programs (e.g., flexible work scheduling and dependent care programs) have positive effects on employees' job satisfaction and further lead to better task performance. Seong (2016) pointed out that those employees who perceive that they are supported by their organizations tend to produce better task performance. Meanwhile, Wang et al. (2013) emphasized a similar conclusion ---- employees will demonstrate more favorable work-related behavior like better task performance if the organization recognize, empathize and identify with their well-being. In conclusion, we may guess that skill variety is likely to be guaranteed and promoted by family friendly, and family friendly in turn leads to better task performance. To replicate this finding, we hypothesize the following:

Hypothesis 2: Family-friendly policies positively mediate the relationship between skill variety and task performance.

\subsection{Market orientation as a moderator}

Although we have hypothesized the positive relationship between skill variety and task performance, the business culture under which this relationship is affected has not been explored adequately. According to Narver and Slater (1990), market orientation is one kind of business culture that produces outstanding performance through its commitment to creating superior value for customers, which consists of three behavioral components ---- customer orientation, competitor orientation and interfunctional coordination. Customer orientation include all of the activities involved in acquiring information about one's target customers and create superior value for them. These activities emphasized the continuous understanding about customer's entire value chain over time. Competitor orientation refers to the understanding of strengths, weaknesses, capabilities and strategies of the key current and key potential competitors. The interfunctional coordination means the coordinated utilization of company resources in creating superior value for target customers. This model is known as the culture-based interpretation of market orientation, which is widely accepted as a root concept in modern marketing theory (Adam, \& Syahputra, 2016).

Market orientation has been repeatedly used as a moderating variable in many literatures (e.g., Hsieh, Tsai, \& Wang, 2008; Pena, Jamilena, \& Molina, 2016). For instance, Pena et al. (2016) suggested that market orientation moderate the relationship between perceived value and customer loyalty. Hsieh et al. (2008) found that market orientation moderate the relationship between product advantage and new product performance, either as a whole or in respect to different types of product performance. Being a business culture, market orientation is likely to exist in various levels of organization and influences the relationship between skill variety and task performance. Kiessling et al. (2015) mentioned that market-oriented firms obtain information and knowledge about their customers' needs and competitors' strategies and then to supply superior offering, these activities require employees to keep learning various skills. Jyoti and Shama (2012) pointed out that market-oriented firms are more likely to recognize the requirements and needs of their employees and hence increase employees' satisfaction. Employees adopting a market orientation may feel that they are making a worthwhile contribution to the firm and in turn create better job performance. Adam and Syahputra (2016) used quantitative method with 200 questionnaires that was spread to 200 managers and showed that market orientation has a positive correlation with business performance. In conclusion, within the market orientation environment, the impact of skill variety and family friendly on task performance may become stronger. Thus, we hypothesize as follows:

Hypothesis 3: Market orientation positively moderate the relationship between skill variety and task performance. 


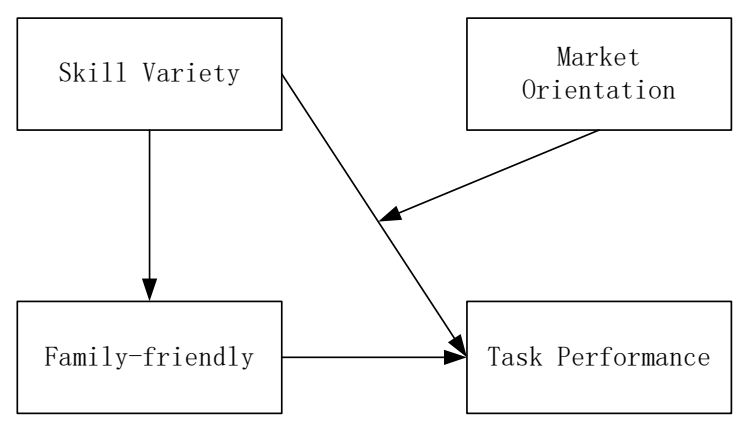

Figure 1. Conceptual model

\section{Participation and procedure}

This study focused on exploring the inherent relations between skill variety, family-friendly and task performance, and test moderating effect of market orientation. In order to verify if this model can be supported in the sample of the China new generation, we used convenience sampling and occasional sampling method and tested the hypotheses with the sample survey.

Because most of the scales used in this study were developed by Western scholars, we had a pilot study before the formal survey to test the items reliability and applicability. 80 questionnaires were distributed and we got 88 percent valid responses with effective rate of 87 percent. Through reliability, validity and project analysis, we adjustor delete some items which are unclear in statement, low reliability or does not meet China's national conditions. Ultimately we form the final scale of this study. The participants in formal study consisted of 525 young employees who were born from 1990-1995 in companies of Fujian, Zhejiang and Guangdong province in China. First we made full use of social networks to set a list of survey target organizations, then had communication with our respondents and confirmed the list; at last we operated the research through interview, mails and e-mail. They come from different industries including: manufactory, construction, transportation, communication, finance, business and hotel, public service and so on.

\section{Measurements}

Skill Variety, Two items from the short form of the Job Characteristics Inventory(JCI; Sims, Szilagyi, \& Keller,1976) were used to assess perceived skill variety. A 5-poit response scale ranging from 1(very little) to 5(a great deal) accompanied the following items: How much variety is there in your job? and To what extent does your job provide the opportunity to do a number of different duties each day? The Cronbach's $\alpha$ for the scale in the current study was 0.80 .

Family-Friendly. FF are employer-sponsored programs and policies that are designed to help employees manage work and personal life demands (Glass \& Finley, 2002; Lobel, 1999). They generally include flexible work schedules, dependent care assistance, leave arrangements, counseling and referral services. In this study, we assess family-friendly on the basis of the FF Search Activities Index, which has a list of 9 FF search activities developed by Hammer et al.(2005). The Cronbach's $\alpha$ for the FF scale in the current study was 0.885 .

Market Orientation. Market orientation, as a corporate culture, characterizes an organization`s disposition of delivering superior value to its customers continuously (Slater and Narver, 1994). This variable is measured by a nine-item scale developed by Han, Jin k. et al. (1998). In this study, the $\alpha$ coefficient for the scale was 0.92 .

Task Performance. The degree of participants' performance creation as a team member was assessed using supervisor ratings of 6 items from Janssen, O. (2008) innovation scale. The response format was the same as the one used for measuring citizenship behavior. Cronbach's alpha was 0.94. 


\section{Results and Discussion}

\subsection{Common method variance analyses}

When self-report questionnaires are used to collect data at the same time from the same participants, common method variance (CMV) may be a concern. We test CMV loads all items from each of the constructs into an exploratory factor analysis to see whether one single factor does emerge or whether one general factor does account for a majority of the covariance between the measures; if not, the claim is that CMV is not a pervasive issue. The expected ex post model showed good fit indexes (RMSEA=0.050; NFI=0.920; CFI=0.951; GFI=0.916; IFI=0.963). Nevertheless, Table 1 presents this model did not yield a significantly better fit to the data than ex ante.

Table 1. Deal with CMV in the ex post statistical analyses.

\begin{tabular}{|c|c|c|}
\hline CMV & Ex-ante & Ex-post \\
\hline$x^{2}$ & 545.133 & 906.436 \\
\hline $\mathrm{df}$ & 535 & 546 \\
\hline$x^{2} / \mathrm{df}$ & 1.010 & 1.660 \\
\hline CFI & 0.996 & 0.951 \\
\hline NFI & 0.943 & 0.920 \\
\hline RMSEA & 0.015 & 0.050 \\
\hline IFI & 0.996 & 0.963 \\
\hline GFI & 0.921 & 0.916 \\
\hline
\end{tabular}

\subsection{Hierarchical regression-mediating effect}

To identify potential mediator of the relationship between skill variety and task performance, three multiple regression models were followed next to test for mediation (Baron and Kenny, 1986). First, a significant relationship was established between skill variety and task performance. Then a significant association was shown between skill variety and family-friendly. At the third step, we use task performance as the criterion variable in a regression equation; both skill variety and family-friendly were entered. The skill variety can still find the significant relations with task performance, but the influence became much weaker, thus partial mediation of family-friendly is indicated, which is shown in Table 2.

Table 2. Mediating multiple regression results.

\begin{tabular}{|c|c|c|c|}
\hline \multirow{2}{*}{ Variables } & \multicolumn{3}{|c|}{ Model 1} \\
\cline { 2 - 4 } & TP & FF & TP \\
\hline Gender & -0.033 & -0.035 & -0.076 \\
\hline Age & 0.032 & -0.009 & -0.027 \\
\hline Education & $0.213^{* * *}$ & 0.051 & $0.222^{* * *}$ \\
\hline Tenure & $0.155^{* * *}$ & 0.075 & $-0.132^{* * *}$ \\
\hline Marital status & -0.080 & 0.015 & 0.008 \\
\hline SV & $0.085^{* * *}$ & $0.235^{* * *}$ & $0.088^{*}$ \\
\hline FF & & & $0.046^{* * *}$ \\
\hline $\mathrm{R}^{2}$ & $0.099^{* * *}$ & $0.133^{* * *}$ & $0.087^{* * *}$ \\
\hline Adj-R & $0.084^{* * *}$ & $0.125^{* * *}$ & $0.075^{* * *}$ \\
\hline $\mathrm{F}$ & $2.986^{* * *}$ & $25.532^{* * *}$ & $1.682^{* * *}$ \\
\hline
\end{tabular}

Notes: Significant at: ${ }^{*} \mathrm{p}<0.05 ;{ }^{* * *} \mathrm{p}<0.001 ; \mathrm{n}=500 ; \mathrm{SV}=$ Skill Variety; FF=Family-friendly; $\mathrm{TP}=$ Task Performance.

\subsection{Hierarchical regression-moderating effect}

As shown above in Table 3, we first test the moderating effect of market orientation between skill variety and task performance from models 2 . In the control of the respondent's gender, age, education, tenure, marital status, the interaction of the preference of skill variety and market orientation is significant $(\beta=0.206, \mathrm{p}<0.01)$; the moderating effect of skill variety and market orientation is 
identified, which means once market clime has stronger preference to chase for task performance, their job effort will lead to stronger fit perception.

Table 3. Moderating hierarchical multiple regression results.

\begin{tabular}{|c|c|c|c|c|}
\hline \multirow{2}{*}{ Variables } & \multicolumn{4}{|c|}{ Model2 } \\
\cline { 2 - 5 } & \multicolumn{4}{|c|}{ TP } \\
\hline Gender & -0.033 & -0.027 & -0.031 & -0.025 \\
\hline Age & 0.032 & -0.028 & -0.025 & -0.018 \\
\hline Education & $0.213^{* * *}$ & $0.022^{* * *}$ & $0.015^{* * *}$ & $0.013^{* * *}$ \\
\hline Tenure & $0.175^{* * *}$ & 0.133 & 0.155 & 0.130 \\
\hline Marital status & -0.080 & 0.024 & 0.018 & 0.015 \\
\hline SV & & $0.228^{* * *}$ & $0.209^{* * *}$ & $0.135^{* * *}$ \\
\hline MAR & & & $0.143^{*}$ & $0.131^{*}$ \\
\hline SV*MAR & & & & $0.206^{* *}$ \\
\hline $\mathrm{R}^{2}$ & $0.099^{* * *}$ & $0.136^{* * *}$ & $0.156^{* * *}$ & $0.182^{* * *}$ \\
\hline Adj-R & $0.084^{* * *}$ & $0.128^{* * *}$ & $0.137^{* * *}$ & $0.163^{* * *}$ \\
\hline $\mathrm{F}$ & $2.986^{* * *}$ & $23.652^{* * *}$ & $23.158^{* * *}$ & $26.149^{* * *}$ \\
\hline
\end{tabular}

Notes: Significance at: ${ }^{*} \mathrm{p}<0.05 ;{ }^{* * *} \mathrm{p}<0.001 ; \mathrm{n}=500$; SV=Skill Variety; FF=Family-friendly; $\mathrm{TP}=$ Task Performance; MAR=Market Orientation; SV*MAR=Skill Variety and market orientation.

\section{Conclusion}

This study extends the literature by examining the consequences of family-friendly on skill variety, market orientation and task performance of employees. As hypothesized, skill variety variables can affect task performance and also has direct and indirect effects on task performance mediated by family-friendly. Moreover, we have deepened and expanded our understanding of the relationship between skill variety and task performance by the moderating effects of market orientation from one aspect to another. In general, this paper contributes to the ongoing efforts to understand the strategic management aspect of family-friendly and job performance.

\section{Limitations and future work}

In the current study, due to resource limitations, all data came from one source,all variables are concerned with perception or self-behaviors, which is difficult to be assessed by others; that may cause single source bias. We need to consider this bias in research design and try to survey team member and their customer in further study to get more accurate data and avoid common variance.

\section{Funding}

This research was supported by Humanity and Social Sciences Research Foundation of Ministry of Education of China (15YJC630069), Sciences Research Talents Project Fund for Outstanding Young Teachers in Fujian Higher Education Institutions,China(2016023).

\section{References}

[1] M. Adam and H. Syahputra, Creating market orientation and business culture to increase SMEs performance in Aceh, Indonesia., DLSU Business \& Economics Review. 26 (2016), 81-91.

[2] T. D. Allen, Family-supportive work environments: The role of organizational perceptions., Journal of Vocational Behavior, 58 (2001), 414-435.

[3] J. G. Caillier, Does satisfaction with family-friendly programs reduce turnover? A panel study conducted in U.S. Federal agencies, Public Personnel Management 45 (2016), 284-307. 
[4] J. P. Campbell, Modeling the performance prediction problem in industrial and organization psychology, Handbook of Industrial and Organization Psychology, Consulting Psychologists Press (1990), 687-732.

[5] C. J. Chen, H. A. Shih and Y. C. Yeh, Individual initiative, skill variety, and creativity: The moderating role of knowledge specificity and creative resources., The International Journal of Human Resource Management. 17 (2011), 3447-3461.

[6] C. H. Chin, M. C. Lo and T. Ramayah, Market orientation and organizational performance: The moderating role of service quality., Sage Open 3 (2013), no. 4, 1-14.

[7] L. C. Chu and C. C. Lai, A research on the influence of leadership style and job characteristics on job performance among accountants of county and city government in Taiwan, Public Personnel Management 40 (2011), 101-118.

[8] K. Daniels, Rethinking job characteristics in work stress research., Human Relations 59 (2006), 267-290.

[9] E. Demerouti, Job characteristics, flow, and performance: The moderating role of conscientio usness., Journal of Occupational Health Psychology 3 (2006), 266-280.

[10] P. Ghosh, A. Rai, R. Chauhan, N. Gupta and A. Singh, Exploring the moderating role of context satisfaction between job characteristics and turnover intention of employees of Indian public sector banks, Journal of Management Development 34 (2015), no. 8, 1019-1030.

[11] G. R. Oldham and H. J, Not what it was and not what it will be: The future of job design research., Journal of Organizational Behavior (2010), no. 31, 463-479.

[12] R. F. Piccolo and J. A. Colquitt, Transformational leadership and job behaviors: The mediating role of job characteristics, Academy of Management Journal 49 (2006), no. 2, 327-340.

[13] R. K. Pradhan, L. K. Jena and I. G. Kumari, Effect of work-life balance on organizational citizenship behaviour: Role of organizational commitment, global business review (2016), no. 17, 155-295. 